\title{
Uncertain Fractional Order Chaotic Systems Tracking Design via Adaptive Hybrid Fuzzy Sliding Mode Control
}

\author{
T.C. Lin, C.H. Kuo, V.E. Balas
}

\section{Tsung-Chih Lin}

Feng-Chia University, 40724, Taichung, Taiwan

E-mail: tclin@fcu.edu.tw

\section{Chia-Hao Kuo}

Ph.D Program in Electrical and Communications Engineering

Feng-Chia University, Taichung, Taiwan

E-mail: peterqo022@hotmail.com

\section{Valentina E. Balas}

Aurel Vlaicu University of Arad, Romania

B-dul Revolutiei 77, 310130 Arad, Romania

E-mail: balas@drbalas.ro

\begin{abstract}
In this paper, in order to achieve tracking performance of uncertain fractional order chaotic systems an adaptive hybrid fuzzy controller is proposed. During the design procedure, a hybrid learning algorithm combining sliding mode control and Lyapunov stability criterion is adopted to tune the free parameters on line by output feedback control law and adaptive law. A weighting factor, which can be adjusted by the trade-off between plant knowledge and control knowledge, is adopted to sum together the control efforts from indirect adaptive fuzzy controller and direct adaptive fuzzy controller. To confirm effectiveness of the proposed control scheme, the fractional order chaotic response system is fully illustrated to track the trajectory generated from the fractional order chaotic drive system. The numerical results show that tracking error and control effort can be made smaller and the proposed hybrid intelligent control structure is more flexible during the design process. Keywords: Fractional order chaotic systems; fuzzy logic control, adaptive hybrid control.
\end{abstract}

\section{Introduction in domain}

Due mainly to its demonstrated applications in numerous seemingly diverse and widespread fields of science and engineering, fractional calculus has gained considerable popularity and importance during past three decades [1]- [2]. In control system, due to the fact that the theoretical aspects are well established, fractional order controllers are successfully used to enhance the performance of the feedback control loop. It is observed that the description of some systems is more accurate when the fractional derivative is used. Nowadays, many fractional-order differential systems behave chaotically, such as the fractional-order Chua's system [3], the fractionalorder Duffing system [4], the fractional-order system, the fractional-order Chen's system [5], the fractional-order cellular neural network [6], the fractional-order neural network [7]. The tracking problem of fractional order chaotic systems is first investigated by Deng and Li [21] who carried out tracking in case of the two fractional Lü systems. Afterwards, they studied chaos tracking of the Chen system with a fractional order in a different manner [22]-[24].

Based on the universal approximation theorem, [9]- [20] (fuzzy logic controllers are general enough to perform any nonlinear control actions) there is rapidly growing interest in systematic 
design methodologies for a class of nonlinear systems using fuzzy adaptive control schemes. Like the conventional adaptive control, the adaptive fuzzy control is classified into direct and indirect fuzzy adaptive control categories [9], [17]- [19]. A direct adaptive fuzzy controller uses fuzzy logic systems as controller in which linguistic fuzzy control rules can be directly incorporated into the controller. On the other hand, an indirect adaptive fuzzy controller uses fuzzy descriptions to model the plant in which fuzzy IF-THEN rules describing the plant can be directly incorporated into the indirect fuzzy controller. Moreover, a hybrid adaptive fuzzy controller can be constructed using a weighting factor to sum together the control efforts from indirect adaptive fuzzy controller and direct adaptive fuzzy controller.

Although the concept of sliding mode control (SMC) and the theory of fractional order system are well known, their integration, fractional sliding mode control, is an interesting filed of research dwelt on this paper with some applications [8]. The motivation of this paper stands on two driving forces: One, most systems in the reality display behavior characterized best in time domain of fractional operators, the other, the uncertainties on the process dynamics can appropriately be alleviated by utilizing SMC technique.

In this paper, by combining the approximate mathematical model, linguistic model description and linguistic control rules into a single adaptive fuzzy controller, an adaptive hybrid fuzzy controller is proposed to achieve prescribed tracking performance of fractional order chaotic systems. A new adaptive hybrid fuzzy SMC algorithm incorporated Lyapunov stability criterion is proposed so that not only the stability of adaptive fuzzy control system is guaranteed but also the influence of the approximation error and external disturbance on the tracking error can be attenuated to an arbitrarily prescribed level.

This paper is organized as follows: In section2, an introduction to fractional derivative and its relation to the approximation solution will be addressed. Section 3 generally proposes adaptive hybrid fuzzy SMC of uncertain fractional order systems in presence of uncertainty and its stability analysis. In Section 4, application of the proposed method on fractional order expression chaotic system is investigated. Finally, the simulation results and conclusion will be presented in Section 5 .

\section{Basic definition and preliminaries for fractional order systems}

The concept of fractional calculus is popularly believed to have steamed from a question raised in the year 1695 by Marquis de L'Hoptial to Gottfried Wilhelm Leibniz. It is a generalization of integration and differentiation to non-integer order fundamental operator, denoted by ${ }_{a} D_{t}^{q}$, where $\mathrm{a}$ and $\mathrm{t}$ are the limits of the operator. This operator is a notation for taking both the fractional integral and functional derivative in a single expression defined as [1]

$$
{ }_{a} D_{t}^{q}= \begin{cases}\frac{d^{q}}{d t^{q}}, & q>0 \\ 1 & q=0 \\ \int_{t}^{a}(d \tau)^{-q}, & q<0\end{cases}
$$

There are some basic definitions for the general fractional and the commonly used definitions are Grunwald-Letnikov and Riemann-Liouville [1]. The Grunwald-Letnikov definition is expressed as

$$
{ }_{a} D_{t}^{q} f(t)=\lim _{h \rightarrow 0} \sum_{j=0}^{\left[\frac{t-a}{h}\right]}(-1)^{j}\left(\begin{array}{l}
a \\
b
\end{array}\right) f(t-j h)
$$


where [.] is the integer part. The simplest and easiest definition is Riemann-Liouville definition given as

$$
{ }_{a} D_{t}^{q} f(t)=\frac{1}{\Gamma(n-q)} \frac{d^{n}}{d t^{n}} \int_{0}^{t} \frac{f(\tau)}{(t-\tau)^{q-n+1}} d \tau
$$

where $n$ is the first integer which is not less q, i.e., $n-1<q<n$, and $\Gamma$ is the Gamma function.

The numerical simulation of a fractional differential equation is not simple as that of an ordinary differential equation. In this paper, the algorithm which is an improved version of Adams-Bashforth-Moulton algorithm to find an approximation for fractional order systems based on predictor-correctors is given. Consider the following differential equation

$$
{ }_{a} D_{t}^{q} y(t)=r(y(t), t), \quad 0 \leq t \leq T \text { and } y^{(k)}(0)=y_{o}^{(k)}, k=0,1,2, \ldots, m-1
$$

where

$$
{ }_{a} D_{t}^{q} y(t)= \begin{cases}\frac{1}{\Gamma(m-q)} \int_{0}^{t} \frac{f^{(m)}(\tau)}{(t-\tau)^{q-m+1}} d \tau, & m-1<q<m \\ \frac{d^{m}}{d t^{m}} y(t), & q=m\end{cases}
$$

and $m$ is the first integer larger the $q$. The solution of the equation (4) is equivalent to Volterra integral equation [1] described as

$$
y(t)=\sum_{k=0}^{[q]-1} y_{0}^{(k)} \frac{t^{k}}{k !}+\frac{1}{\Gamma(q)} \int_{0}^{t}(t-\lambda)^{q-1} r(y(\lambda), \lambda) d \lambda
$$

Let $\mathrm{h}=\mathrm{T} / \mathrm{N}, t_{n}=n h, \mathrm{n}=0,1,2, \cdots \mathrm{N}$. Then (6) can be discretized as follows.

$$
y_{h}\left(t_{n+1}\right)=\sum_{k=0}^{[q]-1} y_{0}^{(k)} \frac{t_{n+1}^{k}}{k !}+\frac{h^{q}}{\Gamma(q+2)} r\left(y_{h}^{p}\left(t_{n+1}\right), t_{n+1}\right)+\frac{h^{q}}{\Gamma(q+2)} \sum_{j=0}^{n} a_{j, n+1} r\left(y_{h}\left(t_{j}\right), t_{j}\right)
$$

where predict value $y_{h}^{p}\left(t_{n+1}\right)$ is determined by

$$
y_{h}^{p}\left(t_{n+1}\right)=\sum_{k=0}^{[q]-1} y_{0}^{(k)} \frac{t_{n+1}^{k}}{k !}+\frac{h^{q}}{\Gamma(q)} \sum_{j=0}^{n} b_{j, n+1} r\left(y_{h}\left(t_{j}\right), t_{j}\right)
$$

and

$$
\begin{gathered}
a_{j, n+1}=\left\{\begin{array}{cl}
n^{q+1}-(n-q)(n+1)^{q}, & j=0 \\
(n-j+2)^{q+1}+(n-j)^{q+1}-2(n-j+1)^{q+1} & 1 \leq j \leq n \\
1 & j=n+1
\end{array}\right. \\
b_{j, n+1}=\frac{h^{q}}{q}\left((n+1-j)^{q}-(n-j)^{q}\right)
\end{gathered}
$$

The approximation error is given as

$$
\max _{j=0,1,2, \cdots N}\left|y\left(t_{j}\right)-y_{h}\left(t_{j}\right)\right|=O\left(h^{p}\right)
$$

where $\mathrm{p}=\min (2,1+\mathrm{q})$. Therefore, the numerical solution of a fraction order chaotic system discussed in this paper can be obtained by applying the above mentioned algorithm. 


\section{Adaptive hybrid fuzzy sliding mode control of uncertain frac- tional order chaotic systems}

In this section, we study adaptive hybrid fuzzy tracking control of uncertain fractional order chaotic systems, i.e., to force output trajectory which is obtained by the algorithm mentioned in section 2 of the response system to track output trajectory of the drive system.

Consider a fractional order chaotic dynamic system

$$
x^{(n q)}=f(\underline{x}, t)+g(\underline{x}, t) u+d(t), \quad y=x_{1}
$$

where $\underline{x}=\left[x_{1}, x_{2}, \ldots, x_{n}\right]^{T}=\left[x, x^{(q)}, x^{(2 q)}, \ldots, x^{((n-1) q)}\right]^{T}$ is the state vector, $f(\underline{x}, t)$ and $g(\underline{x}, t)$ are unknown but bounded nonlinear functions which express system dynamics, $d(t)$ is the external bounded disturbance, $|d(t)| \leq D$, and $\mathrm{u}(\mathrm{t})$ is the control input. The control objective is to force the system output $y$ to follow a bounded reference signal $y_{d}$ which is the output trajectory of a drive system, under the constraint that all signals involved must be bounded. To begin with, the reference signal vector $\underline{y}_{d}$ and the tracking error vector $\underline{e}$ will be defined as

$$
\begin{gathered}
\underline{y}_{d}=\left[y_{d}, y_{d}^{(q)}, \ldots, y_{d}^{((n-1) q)}\right]^{T} \in R^{n}, \\
\underline{e}=\underline{y}_{d}-\underline{x}=\left[e, e^{(q)}, e^{(2 q)}, \ldots, e^{((n-1) q)}\right]^{T} \in R^{n}, e^{(i q)}=y_{d}^{(i q)}-x^{(i q)}=y_{d}^{(i q)}-y^{(i q)}
\end{gathered}
$$

In general, in the space of the error state a sliding surface is defined by

$$
s(\underline{x}, t)=-(\underline{k e})=-\left(k_{1} e+k_{2} e^{(q)}+\ldots+k_{n-1} e^{(n-2) q}+e^{(n-1) q}\right)
$$

where $\underline{\underline{k}}=\left[k_{1}, k_{2}, \ldots, k_{n-1}, 1\right]$ in which the $k_{i}$ 's are all real and are chosen such that $h(r)=$ $\sum_{i=1}^{n} k_{i} r^{(i-1) q}, k_{n}=1$ is a Hurwitz polynomial where $r$ is a Laplace operator. The tracking problem will be considered as the state error vector $\underline{e}$ remaining on the sliding surface $s(\underline{x}, t)=0$ for all $t \geq 0$. The sliding mode control process can be classified into two phases, the approaching phase with $s(\underline{x}, t) \neq 0$ and the sliding phase with $\mathrm{s}(\underline{\mathrm{x}}, \mathrm{t})=0$ for initial error $\underline{e}(0)=0$. In order to guarantee that the trajectory of the state error vector $\underline{e}$ will translate from the approaching phase to the sliding phase, the sufficient condition

$$
s(\underline{x}, t) \dot{s}(\underline{x}, t) \leq-\eta>0
$$

must be satisfied. Two type of control law must be derived separately for those two phases described above. In the sliding phase, it implies $s(\underline{x}, t)=0$ and $s^{(q)}(\underline{x}, t)=0$. In order to force the system dynamics to stay on the sliding surface, the equivalent control $\bar{u}$ can be derived as follows:

If $f(\underline{x}, t)$ and $g(\underline{x}, t)$ are known and free of external disturbance, i.e., $\mathrm{d}(\mathrm{t})=0$, taking the derivative of the sliding surface with respective to time, we get

$$
\begin{array}{r}
s^{(q)}=-\left(\sum_{i=1}^{n-1} c_{i} e^{(i q)}+e^{(n q)}\right)=-\left(\sum_{i=1}^{n-1} k_{i} e^{(i q)}+y_{d}^{(n q)-y^{(n q)}}\right) \\
=-\left(\sum_{i=1}^{n-1} k_{i} e^{(i q)-f(\underline{x}, t)-g(\underline{x}, t)} u_{e q}\right)-y_{d}^{(n)}=-\sum_{i=1}^{n-1} k_{i} e^{(i)}+f(\underline{x})+b(\underline{x}) \bar{u}(t)-x_{d}^{(n)}=0
\end{array}
$$

Therefore, the equivalent control can be obtained as 


$$
\bar{u}=\frac{1}{g(\underline{x}, t)}\left(\sum_{i=1}^{n-1} k_{i} e^{(i q)}-f(\underline{x}, t)+y_{d}^{(n q)}\right)
$$

On the contrary, in the approaching phase, $s(\underline{x}, t) \neq 0$, an approaching-type control $u_{a p}$ must be added in order satisfy the sufficient condition (4) and the complete sliding mode control will be expressed as

$$
u=\bar{u}-u_{a p}, \quad u_{a p}=\psi_{h} \operatorname{sgn}(s)
$$

where $\psi_{h} \geq \eta>0$.

To obtain the sliding mode control (17), the system functions $f(\underline{x}, t), g(\underline{x}, t)$ and switching parameter $\psi_{h}$ must be known in advance. However, $f(\underline{x}, t)$ and $g(\underline{x}, t)$ are unknown and external disturbance, $d(t) \neq 0$, the ideal control effort (16) cannot be implemented. We replace $f(\underline{x}, t)$, $g(\underline{x}, t)$ and $u_{a p}$ by the fuzzy logic system $f\left(\underline{x} \mid \underline{\theta}_{f}\right), g\left(\underline{y} \mid \underline{\theta}_{g}\right)$ and $h\left(s \mid \theta_{h}\right)$ in specified form as [9], [17][19], i.e.,

$$
f\left(\underline{x} \mid \underline{\theta}_{f}\right)=\xi^{T}(\underline{x}) \underline{\theta}_{f}, g\left(\underline{x} \mid \underline{\theta}_{g}\right)=\xi^{T}(\underline{x}) \underline{\theta}_{g}, \quad h\left(s \mid \underline{\theta}_{h}\right)=\emptyset^{T}(s) \underline{\theta}_{h}
$$

let $\left|h\left(s \mid \underline{\theta}_{h}\right)\right|=D+\psi_{h}+\omega_{\max }$ when $s(\underline{x}, t)$ is outside the boundary layer. Here the fuzzy basis functions $\xi(\underline{x})$ and $\emptyset(s)$ depend on the fuzzy membership functions and is supposed to be fixed, while $\theta_{f}, \theta_{g}$ and $\theta_{h}$ are adjusted by adaptive laws based on Lyapunov stability criterion. Therefore, depending on plant knowledge and control knowledge, a hybrid adaptive fuzzy controller can be constructed by incorporating both fuzzy description and fuzzy control rules using a weighting factor $\alpha$ to combine the indirect adaptive fuzzy controller and the direct adaptive fuzzy controller. Based on the trade-off between plant knowledge and control knowledge, the weighting factor $\alpha \in[1,1]$ can be adjusted. Therefore, the total control effort can be expressed as

$$
u_{c}=\alpha u_{i}+(1-\alpha) u_{d}
$$

where the direct adaptive fuzzy controller $u_{d}$ and the indirect adaptive fuzzy controller $u_{i}$ are given as follows:

$$
u_{d}(\underline{x})=u_{D}\left(\underline{x} \mid \underline{\theta}_{D}\right)-\frac{h\left(s \mid \underline{\theta}_{h}\right)}{g(\underline{x}, t)} \text { and } u_{i}(\underline{x})=\frac{1}{g\left(\underline{x} \mid \underline{\theta}_{g}\right)}\left[\sum_{i=1}^{n-1} k_{i} e^{(i q)}+y_{d}^{(n q)}-f\left(\underline{x}, \mid \underline{\theta}_{f}\right)-h\left(s \mid \underline{\theta}_{h}\right)\right]
$$

where $u_{D}(\underline{x} \mid \underline{\theta})$ is obtained by fuzzy logic system specified as

$$
u_{D}\left(\underline{x} \mid \underline{\theta}_{D}\right)=\xi^{T}(\underline{x}) \underline{\theta}_{D}
$$

The optimal parameter estimations $\underline{\theta}_{f}^{*}, \underline{\theta}_{g}^{*}, \underline{\theta}_{h}^{*}$ and $\underline{\theta}_{D}^{*}$ are defined as

$$
\begin{gathered}
\underline{\theta}_{f}^{*}=\arg \min _{\underline{\theta}_{f} \in \Omega_{f}}\left[\sup _{x \in \Omega_{x}}\left|f\left(\underline{x} \mid \underline{\theta}_{f}\right)-f(\underline{x}, t)\right|\right], \underline{\theta}_{g}^{*}=\arg \min _{\underline{\theta}_{g} \in \Omega_{g}}\left[\sup _{x \in \Omega_{x}}\left|g\left(\underline{x} \mid \underline{\theta}_{g}\right)-g(\underline{x}, t)\right|\right] \\
\underline{\theta}_{D}^{*}=\arg \min _{\underline{\theta}_{D} \in \Omega_{D}}\left[\sup _{x \in \Omega_{x}}\left|u_{D}\left(\underline{x} \mid \underline{\theta}_{g}\right)-\bar{u}\right|\right], \underline{\theta}_{h}^{*}=\arg \min _{\underline{\theta}_{h} \in \Omega_{h}}\left[\sup _{\underline{x} \in \Omega_{\underline{x}}}\left|h\left(s \mid \underline{\theta}_{h}\right)-u_{a p}\right|\right]
\end{gathered}
$$


where $\Omega_{f}, \Omega_{g}, \Omega_{D}$ and $\Omega_{\underline{x}}$ are constraint sets of suitable bounds on $\underline{\theta}_{f}, \underline{\theta}_{g}, \underline{\theta}_{h}^{*}, \underline{\theta}_{D}$ and x respectively and they are defined as $\Omega_{f}=\left\{\underline{\theta}_{f}\left|\underline{\theta}_{f}\right| \leq M_{f}\right\}, \Omega_{g}=\left\{\underline{\theta}_{g}\left|\underline{\theta}_{g}\right| \leq M_{g}\right\}, \Omega_{D}=\left\{\underline{\theta}_{D}\left|\underline{\theta}_{D}\right| \leq M_{D}\right\}$, $\Omega_{h}=\left\{\underline{\theta}_{h}\left|\underline{\theta}_{h}\right| \leq M_{h}\right\}$ and $\Omega_{x}=\left\{x|| x \mid \leq M_{x}\right\}$, where $M_{f}, M_{g}, M_{D}, M_{h}$ and are positive constants.

By using (20), (21), sliding surface equation (15) can be rewritten as

$$
\begin{gathered}
s^{(q)}=\omega+\alpha\left[f\left(\underline{x} \mid \underline{\theta}_{f}^{*}\right)-f\left(\underline{x} \mid \underline{\theta}_{f}\right)\right]+\alpha\left[g\left(\underline{x} \mid \underline{\theta}_{g}^{*}\right)-g\left(\underline{x} \mid \underline{\theta}_{g}\right)\right] u_{i}-(1-\alpha) h\left(\underline{s} \mid \underline{\theta}_{h}^{*}\right) \\
-\alpha h\left(\underline{s} \mid \underline{\theta}_{h}\right)-(1-\alpha) g(\underline{x})\left[u_{D}\left(\underline{x} \mid \underline{\theta}_{D}^{*}\right)-u_{D}\left(\underline{x} \mid \underline{\theta}_{D}\right)\right]+\alpha h\left(\underline{s} \mid \underline{\theta}_{h}^{*}\right)-\alpha h\left(\underline{s} \mid \underline{\theta}_{h}^{*}\right) \\
+(1-\alpha) h\left(\underline{s} \mid \underline{\theta}_{h}^{*}\right)-(1-\alpha) h\left(\underline{s} \mid \underline{\theta}_{h}^{*}\right)+d(t)
\end{gathered}
$$

where the minimum approximation errors is defined as

$$
\omega=\alpha\left[f(\underline{x})-f\left(\underline{x} \mid \underline{\theta}_{f}^{*}\right)\right]+\alpha\left[g(\underline{x})-g\left(\underline{x} \mid \underline{\theta}_{g}^{*}\right)\right] u_{i}+(1-\alpha)\left[u_{D}\left(\underline{x} \mid \underline{\theta}^{*}\right)-u_{D}\right]
$$

If $\underline{\tilde{\theta}}_{f}=\underline{\theta}_{f}-\underline{\theta}_{f}^{*}, \underline{\tilde{\theta}}_{g}=\underline{\theta}_{g}-\underline{\theta}_{g}^{*}$ and, $\underline{\tilde{\theta}}_{D}=\underline{\theta}_{D}-\underline{\theta}_{D}^{*}$, we have

$$
\begin{aligned}
s^{(q)}= & -(1-\alpha) h\left(\underline{s} \mid \underline{\theta}_{h}^{*}\right)+\omega-\alpha \underline{\tilde{\theta}}_{h}^{T} \emptyset(s)-\alpha \underline{\tilde{\theta}}_{f}^{T} \xi(\underline{x})-\alpha \underline{\tilde{\theta}}_{g}^{T} \xi(\underline{x}) u_{i} \\
& +(1-\alpha) g(\underline{x}) \underline{\tilde{\theta}}_{D}^{T} \xi(\underline{x})-\alpha h\left(\underline{s}^{\prime} \underline{\theta}_{h}^{*}\right)-(1-\alpha) \underline{\tilde{\theta}}_{h}^{T} \emptyset+d(t)
\end{aligned}
$$

Following the proceeding consideration, the following theorem can be obtained.

Theorem: Consider the fractional order SISO nonlinear chaotic system (12) with control input (19), if the fuzzy-based adaptive laws are chosen as

$$
\theta_{f}^{(q)}=r_{1} s \xi(\underline{x}), \quad \theta_{g}^{(q)}=r_{2} s \xi(\underline{x}) u_{i}, \quad \theta_{D}^{(q)}=r_{3} s \emptyset(\underline{s}) \text { and } \theta_{h}^{(q)}=-r_{4} s g(\underline{x}) \xi(\underline{x})
$$

where $r_{i}>0, i=1 \sim 4$. Then, the overall adaptive scheme guarantees the global stability of the resulting closed-loop system in the sense that all signals involved are uniformly bounded and the tracking error will converge to zero asymptotically.

Proof: In order to analyze the closed-loop stability, the Lyapunov function candidate is chosen as

$$
V=\frac{1}{2} s^{2}+\frac{\alpha}{2 r_{1}} \underline{\tilde{\theta}}_{f}^{T} \underline{\tilde{\theta}}_{f}+\frac{\alpha}{2 r_{2}} \underline{\tilde{\theta}}_{g}^{T} \tilde{\tilde{\theta}}_{g}+\frac{\alpha}{2 r_{4}} \underline{\tilde{\theta}}_{h}^{T} \underline{\tilde{\theta}}_{h}+\frac{(1-\alpha)}{2 r_{3}} \underline{\tilde{\theta}}_{D}^{T} \underline{\tilde{\theta}}_{D} \frac{(1-\alpha)}{2 r_{4}} \underline{\hat{\theta}}_{h}^{T} \underline{\tilde{\theta}}_{h}
$$

Taking the derivative of the (26) with respect to time, we get

$$
\begin{aligned}
& V^{(q)}=s s^{(q)}+\frac{\alpha}{r_{1}} \underline{\tilde{\theta}}_{f}^{T} \tilde{\tilde{\theta}}_{f}^{(q)}+\frac{\alpha}{r_{2}} \underline{\tilde{\theta}}_{g}^{T} \tilde{\tilde{\theta}}_{g}^{(q)}+\frac{\alpha}{r_{4}} \underline{\tilde{\theta}}_{h}^{T} \underline{\tilde{\theta}}_{h}^{(q)}+\frac{(1-\alpha)}{r_{3}} \underline{\tilde{\theta}}^{T} \underline{\tilde{\theta}}^{q}+\frac{(1-\alpha)}{r_{4}} \underline{\tilde{\theta}}_{h}^{T} \tilde{\theta}_{h}^{(q)} \\
& =-(1-\alpha) \operatorname{sh}\left(\underline{s} \underline{\theta}_{h}^{*}\right)+s \omega-\alpha s \underline{\tilde{\theta}}_{h}^{T} \emptyset(s)-\alpha s \underline{\tilde{\theta}}_{h}^{T} \xi(\underline{x})-\alpha s \underline{\tilde{\theta}}_{g}^{T} \xi(\underline{x}) u_{i}+(1-\alpha) s g\left(\underline{x}^{\prime} \underline{\tilde{\theta}}^{T} \xi(\underline{x})\right. \\
& -\operatorname{ash}\left(\underline{s} \underline{\theta}_{h}^{*}\right)-(1-\alpha) s \underline{\tilde{\theta}}_{h}^{T} \emptyset+s d(t)+\frac{\alpha}{r_{1}} \underline{\tilde{\theta}}_{f}^{T} \tilde{\tilde{\theta}}_{f}^{(q)}+\frac{\alpha}{r_{2}} \underline{\tilde{\theta}}_{g}^{T} \tilde{\tilde{\theta}}_{g}^{(q)}+\frac{\alpha}{r_{4}} \underline{\tilde{\theta}}_{h}^{T} \tilde{\tilde{\theta}}_{h}^{(q)}+\frac{(1-\alpha)}{r_{3}} \underline{\tilde{\theta}}^{T} \underline{\tilde{\theta}}^{q}+\frac{(1-\alpha)}{r_{4}} \underline{\hat{\theta}}_{h}^{T} \underline{\tilde{\theta}}_{h}^{(q)} \\
& \leq \frac{\alpha}{r_{1}} \underline{\tilde{\theta}}_{f}^{T}\left(\underline{\tilde{\theta}}_{f}^{(q)}-r_{1} s \xi(\underline{x})\right)+\frac{\alpha}{r_{2}} \tilde{\tilde{\theta}}_{g}^{T}\left(\underline{\tilde{\theta}}_{g}^{(q)}-r_{2} s \xi(\underline{x}) u_{i}\right)+\frac{1}{r_{4}} \underline{\tilde{\theta}}_{h}^{T}\left(\underline{\tilde{\theta}}_{h}^{(q)}-r_{4} s \emptyset(\underline{s})\right)-\alpha s(D+\eta) \operatorname{sgn}(s) \\
& +\frac{1-\alpha}{r_{3}} \underline{\tilde{\theta}}^{T}\left(\underline{\tilde{\theta}}^{(q)}+r_{3} s g(\underline{x}) \xi(\underline{x})\right)-(1-\alpha) s\left(D+\psi_{h}\right) \operatorname{sgn}(s)+s d(t)+s \omega
\end{aligned}
$$

From the robust compensator $u_{a}$ and the fuzzy-based adaptive laws are given (25), after simple manipulation, we have

$$
V^{(q)} \leq s \omega-s \psi_{h} \operatorname{sgn}(s)=s \omega-|s| \psi_{h}
$$

Using the corollary of Barbalat's Lemma [16]-[19], we have $\lim _{t \rightarrow \infty}|s(\underline{x}, t)|=0$. Therefore, $\lim _{t \rightarrow \infty}|e(t)|=0$. The proof is completed. 


\section{Simulation example}

In this section, we will apply our adaptive hybrid fuzzy sliding mode controller to force the fractional order chaotic gyro response system to track the trajectory of the fractional order chaotic gyro drive system.

Example: The fractional order chaotic gyro drive and response systems are given as follows:

Drive System:

$$
\left\{\begin{array}{l}
y_{1}^{(q)}=y_{2} \\
y_{2}^{(q)}=-100\left(\frac{y_{1}}{4}\right)+\frac{y_{1}^{3}}{12}-0.5 y_{2}-0.05 y_{2}^{3}+\sin \left(y_{1}\right)+35.5 \sin (2 t) y_{1}-\frac{x_{1}^{3}}{6}+d(t)
\end{array}\right.
$$

Response System:

$$
\left\{\begin{array}{l}
x_{1}^{(q)}=x_{2} \\
x_{2}^{(q)}=-100\left(\frac{x_{1}}{4}\right)+\frac{x_{1}^{3}}{12}-0.7 x_{2}-0.08 x_{2}^{3}+\sin \left(x_{1}\right)+33 \sin (2 t) x_{1}-\frac{x_{1}^{3}}{6}+\Delta f\left(x_{1}, x_{2}\right)+d(t)+u_{c}(t)
\end{array}\right.
$$

where structured uncertainty $\Delta f\left(x_{1}, x_{2}\right)=-0.1 \sin \left(x_{1}\right)$ and external disturbance $d(t)=$ $0.2 \cos (\pi t)$. The main objective is to control the trajectories of the response system to track the reference trajectories obtained from the drive system. The initial conditions of drive and response systems are chosen as $\left[y_{1}(0), y_{2}(0)\right]^{T}=[1,-1]^{T}$ and $\left[x_{1}(0), x_{2}(0)\right]^{T}=[1.6,0.8]^{T}$, respectively. For $\mathrm{q}=0.95, \alpha=0.7$ and all design constants are specified as $k_{1}=k_{2}=1, r_{1}=150, r_{2}=20, r_{3}=$ $1, r_{4}=1$ and step size $h=0.01$. The phase portrait of the drive and response systems for free of control input is given in Figure 1. It is obvious that the tracking performance is bad without control effort supplied to response system.

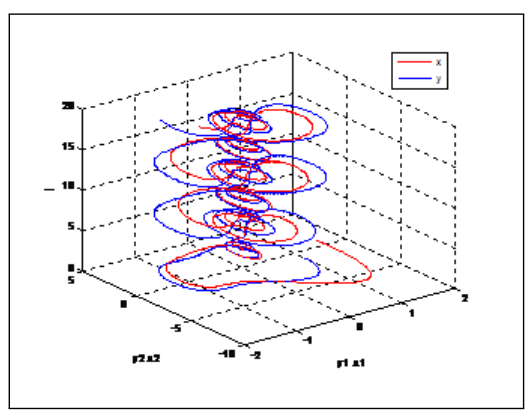

Figure 1: Phase portrait of chaotic drive and response systems

The membership functions for $x_{i} \mathrm{i}=1,2$ are selected as follows:

$$
\begin{aligned}
& \mu_{F_{1}^{i}}\left(x_{i}\right)=\exp \left[-0.5\left(\frac{x_{i}-4}{2}\right)^{2}\right], \mu_{F_{2}^{i}}\left(x_{i}\right)=\exp \left[-0.5\left(\frac{x_{i}-2.7}{2}\right)^{2}\right], \mu_{F_{3}^{i}}\left(x_{i}\right)=\exp \left[-0.5\left(\frac{x_{i}-1.2}{2}\right)^{2}\right], \\
& \mu_{F_{4}^{i}}\left(x_{i}\right)=\exp \left[-0.5\left(\frac{x_{i}}{2}\right)^{2}\right], \mu_{F_{5}^{i}}\left(x_{i}\right)=\exp \left[-0.5\left(\frac{x_{i}+1.2}{2}\right)^{2}\right], \mu_{F_{6}^{i}}\left(x_{i}\right)=\exp \left[-0.5\left(\frac{x_{i}+2.7}{2}\right)^{2}\right], \\
& \mu_{F_{7}^{i}}\left(x_{i}\right)=\exp \left[-0.5\left(\frac{x_{i}+4}{2}\right)^{2}\right],
\end{aligned}
$$

From the adaptive laws (25)-(28), the control effort of the response system can be obtained as

$$
u_{c}=\alpha u_{i}+(1-\alpha) u_{d}
$$

Figure 2 shows the trajectories of the states $x_{i}, y_{i}$ and $x_{2}, y_{2}$, respectively. Control effort trajectory is given in Figure 3 and phase portrait, tracking performance, of the drive and response 


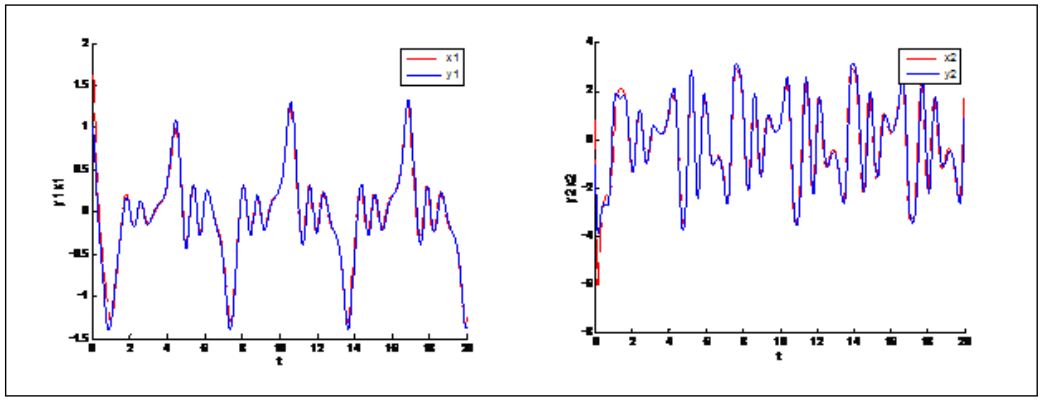

Figure 2: The trajectories of the states $x_{i}, y_{i}$ and $x_{2}, y_{2}$

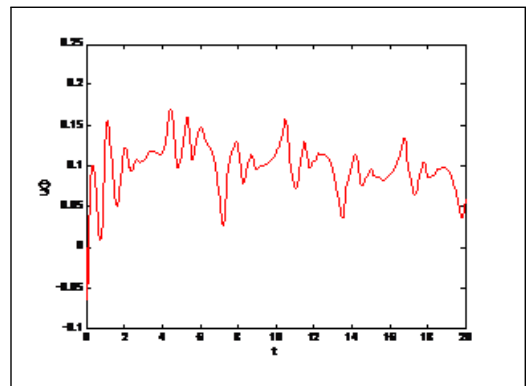

Figure 3: Trajectory of the control effort

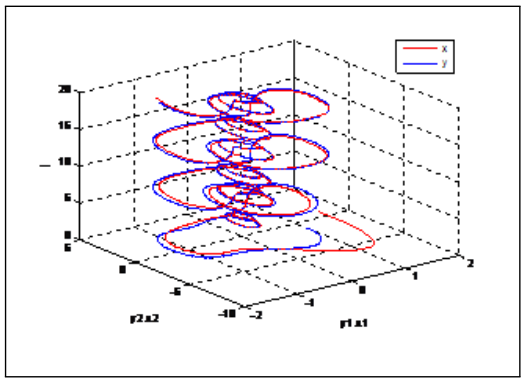

Figure 4: Phase portrait, tracking performance, of the drive and response systems

systems is shown in Figure 4. Trajectory of the sliding surface is given is Figure 5. The maximum value of $V^{(q)}(t)$ is $-1.711 \mathrm{e}-4$ which is always negative defined and consequently is stable.

In order to show the robustness of the proposed adaptive hybrid fuzzy sliding mode control, the control effort is activated at 5 second. The phase portrait, tracking performance, of the drive and response systems is given in Figure 6. Figure 7 shows the trajectories of the states $x_{i}, y_{i}$ and $x_{2}, y_{2}$ respectively. We can see that a fast tracking of drive and response is achieved as the control effort is activated. Control effort trajectory is given in Figure 8. Trajectory of the sliding surface is given is Figure 9. The maximum value of $V^{(q)}(t)$ is $-1.732 \mathrm{e}-4$ which is always negative defined and consequently is stable.

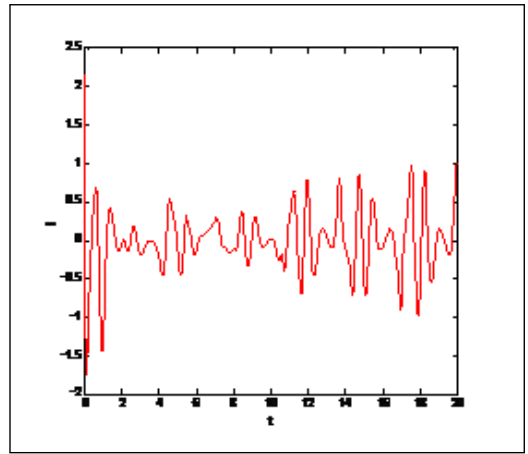

Figure 5: Trajectory of the sliding surface

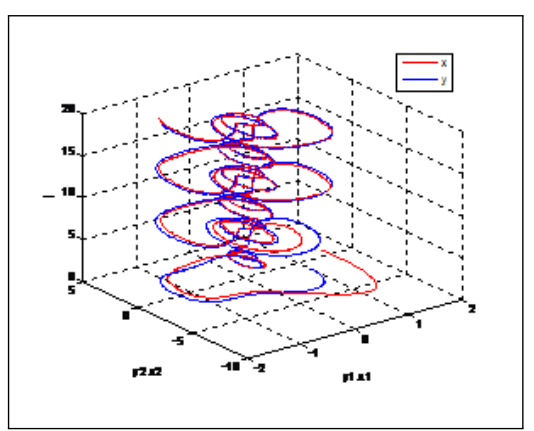

Figure 6: Phase portrait, tracking performance, of the drive and response systems 

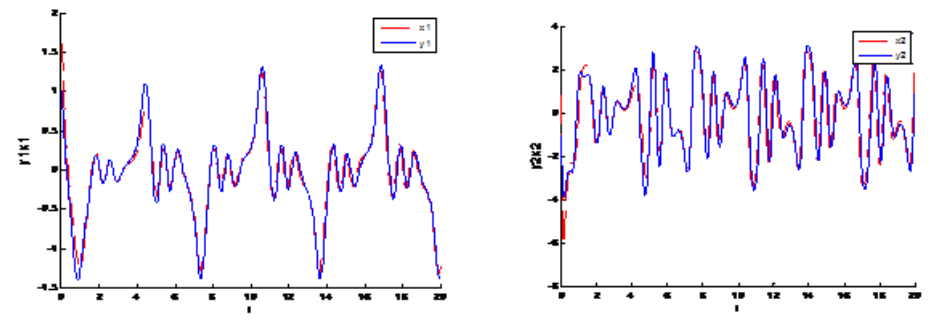

Figure 7: The trajectories of the states $x_{i}, y_{i}$ and $x_{2}, y_{2}$

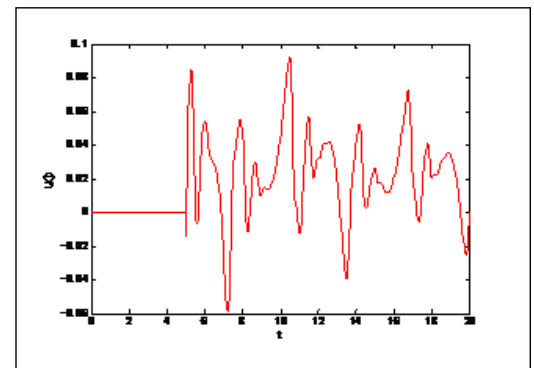

Figure 8: Trajectory of the control effort

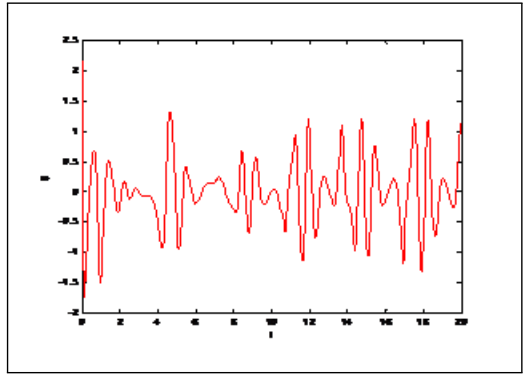

Figure 9: Trajectory of the sliding surface

\section{Conclusions}

A novel adaptive hybrid fuzzy sliding mode controller is proposed to achieve tracking performance of fractional order chaotic systems in this paper. It is a flexible design methodology by the trade-off between plant knowledge and control knowledge using a weighting factor ? adopted to sum together the control effort from indirect adaptive fuzzy controller and direct adaptive fuzzy controller. Based on the Lyapunov synthesis approach, free parameters of the adaptive fuzzy controller can be tuned on line by output feedback control law and adaptive laws. The simulation example, the output trajectory of the fractional order chaotic response system to tracking the trajectory of the fractional order chaotic drive system, is given to demonstrate the effectiveness of the proposed methodology.

\section{Bibliography}

[1] M. S. Tavazoei, M. Haeri, Synchronization of chaotic fractional-order systems via active sliding mode controller, Physica A, Vol. 387, pp. 57-70, 2008.

[2] A.A. Kilbas, H.M. Srivastava and J.J. Trujillo, Theory and applications of fractional differential equations, North-Holland Math. Studies 204, Elsevier, Amsterdam, 2006.

[3] I. Petras, A note on the fractional-order Chua's system, Chaos, Solitons \& Fractals, 38 (1), pp. 140-14,7 2008.

[4] X. Gao and J. Yu, Chaos in the fractional order periodically forced complex Duffing's oscillators, Chaos, Solitons \& Fractals 26, pp. 1125-1133, 2005.

[5] J.G. Lu and G. Chen, A note on the fractional-order Chen system, Chaos, Solitons \& Fractals 27, pp. 685-688, 2006. 
[6] P. Arena and R. Caponetto, Bifurcation and chaos in non-integer order cellular neural networks, Int J Bifurcat Chaos 8 (7), pp. 1527-1539, 1998.

[7] Petras I., A note on the fractional-order cellular neural networks. In: Proceedings of the IEEE world congress on computational intelligence, international joint conference on neural networks, Vancouver, Canada; pp.16-21, 2006.

[8] S. H. Hosseninnia, R. Ghaderi, A. Ranjbar N., M. Mahmoudian, S. Momani, Sliding mode synchronization of an uncertain fractional order chaotic system, Journal Computers \& Mathematics with Applications, Vol. 59, pp. 1637-1643, 2010.

[9] L.A. Zadeh, Fuzzy logic, neural networks and soft computing. Commun. ACM 37 3, pp. 77-84, 1994.

[10] X. Z. Zhang; Y.N. Wang; X. F. Yuan, H? Robust T-S Fuzzy Design for Uncertain Nonlinear Systems with State Delays Based on Sliding Mode Control, International Journal of Computers Communications \& Control, 5(4):592-602, 2010.

[11] Balas, M.M., Balas, V.E., World Knowledge for Control Applications by Fuzzy-Interpolative Systems, International Journal of Computers Communications and Control, Vol. 3, Supplement: Suppl. S, pp. 28-32, 2008.

[12] L. X. Wang, and J. M. Mendel, Fuzzy basis function, universal approximation, and orthogonal least square learning, IEEE Trans. Neural Networks, vol. 3, no. 5, pp. 807-814, 1992.

[13] L. X. Wang, Adaptive Fuzzy Systems and Control: Design and Stability Analysis. Englewood Cliffs, NJ: Prentice-Hall, 1994.

[14] K. Diethelm, An algorithm for the numerical solution of differential equations of fractional order, Elec. Trans. Numer. Anal. 5, pp. 1-6, 1997.

[15] J. L. Castro, "Fuzzy logical controllers are universal approximators," IEEE Trans. Syst., Man, Cybern., 25, pp. 629-635, 1995.

[16] S. S. Ge, T. H. Lee, C. J. Harris, Adaptive Neural Network Control of Robotic Manipulators, World Scientific Publishing Co., Singapore, 1998.

[17] C. H. Wang, T. C. Lin, T. T.. Lee and H. L. Liu, "Adaptive Hybrid Intelligent Control for Unknown Nonlinear Dynamical Systems", IEEE Transaction on Systems, Man, and Cybernetics Part B, 32 (5), October, pp. 583-597, 2002.

[18] C. H. Wang, H. L. Liu and T. C. Lin, "Direct Adaptive Fuzzy-Neural Control with Observer and Supervisory Control for Unknown Nonlinear Systems", IEEE Transaction on Fuzzy Systems, 10(1), pp. 39-49, 2002.

[19] T. C. Lin, C. H. Wang and H. L. Liu, "Observer-based Indirect Adaptive Fuzzy-Neural Tracking Control for Nonlinear SISO Systems Using VSS and ", Fuzzy Sets and Systems, 143, pp.211-232, 2004.

[20] L. A. Zadeh, Knowledge Representation in Fuzzy Logic, IEEE Trans. Knowledge and Data Engineering, 1 (1), pp. 89-100, 1989. 